\title{
TRATAMIENTO DE LA DIARREA AGUDA GRAVE Y TOXICOSIS DEL LACTANTE EN EL HOSPITAL DE ARICA
}

\author{
Dr. GUILLERMO BRAND y Dra. AMELIA DEL VILLAR \\ Servicio de Pediatría. Hospital de Arica
}

\section{GENERALIDADES}

La diarrea aguda es una de las afecciones más frecuentes en el lactante y es la causa principal de mortalidad infantil en nuestro medio. Según Meneghello" "no menos de un tercio de las muertes entre el primer año de vida se determinan di recta o indirectamente por síndromes diarreicos agudos". En 1955 fallecieron en la República de Chile 28.320 menores de un año. Si nos atenemos a lo dicho por Meneghello, debemos aceptar que han fallecido más de 9.000 niños menores de un año, por diarrea.

En un estudio realizado por nosotros en la ciudad de Arica*, se pudo apreciar que durante 1956 fallecieron 100 niños menores de un año, de los cuales en $37(37 \%)$, la causa primitiva fué la diarrea. En ese mismo estudio concluímos que el $84 \%$ de los fallecidos por diarrea eran distróficos y el $90 \%$ eran niños sin un control pediátrico eficiente.

Resaltan a la vista las dos últimas cifras. Mientras no se disponga de un control pediátrico eficiente de nuestros ninos proletarios que nos permita contar con una mayoría de eutróficos capaces de afrontar las inclemencias del medio en que viven, debemos seguir combatiendo en la sala de hospital los cuadros de diarrea grave y su complicación más temible: la toxicosis.

El tratamiento de la diarrea grave y toxicosis del lactante descansa sobre tres pilares fundamentales, los cuales deben coordinarse en forma perfecta para obtener el mayor beneficio posible. Los analizaremos detalladamente:

\section{A. Tratamiento Antibiótico.}

Actualmente se acepta que la gran mayoría de estas diarreas son de origen infeccioso 4 . Los últimos estudios bacteriológicos de deposiciones ${ }^{4 . t}$, permiten concluir que se ha identificado un nuevo germen causante de diarreas. Se trata de la Escherichia Coli Enteropatógena, de la cual existen varias cepas. Se calcula que en más de la mitad de los pacientes este germen es el causante del cuadro diarreico. Las Salmonellas y Shigellas ya no aparecen con la frecuencia con que lo hacían antes.

El antibiótico de elección continúa siendo el Cloramfenicol en dosis de 50 mgrs. por kilo de peso. Autores extranjeros" afirman que la Neomicina por vía enteral sería la droga de elección contra la Escherichia Coli Enteropatógena.

\section{B. Tratamiento Dietético.}

El tratamiento dietético que continúa a la hidratación debe hacerse con leches curativas (Eledón o Nestalba), concentrándolas en forma progresiva y administrándolas cada 2 o 3 horas.

\section{Tratamiento Hidratante.}

Consideramos al tratamiento hidratante como el más importante, para corregir los grandes trastornos del metabolismo hidrosalino con que ingresan nuestros pacientes.

Las profundas alteraciones metabólicas que se producen en el lactante que padece de una diarrea intensa, y en especial cuando ésta se acompaña de vómitos, no sólo derivan de la gran pérdida de agua y electrólitos, sino también de la disminución del aporte energético ${ }^{\top}$. Se produce una alteración tan profunda en el metabolismo celular comprometiendo a los diferentes órganos de la economía y en especial al sistema nervioso, que termina con la muerte del paciente si no se interviene rápidamente administrando en la forma más equilibrada posible los elementos que se encuentran en déficit.

El tratamiento de la deshidratación aguda consistirá en reponer el agua perdida, los electrólitos y administrar elementos energéticos. El agua se administrará en cantidad de $200 \mathrm{cc}$. por kilo de peso en las primeras 24 horas y posteriormente según la evolución clínica del paciente. Los electrólitos deberán administrarse tratando de reponer en la forma más completa posible los que se han perdido por diarrea y vómito. En cuanto a la ración 
energética, se administrarán hidratos de carbono, principalmente glucosa.

La vía de elección para tratar la deshidratación aguda grave, es la endovenosa. $\mathrm{Si}$ ésta no es posible, deberá recurrirse a la gastroclisis.

Nos parece del mayor interés referimos a la inclusión del potasio en las soluciones hidratantes. Este electrólito efectúa múltiples e importantes funciones en el organismo*. Entre ellas destaca su intervención en el metabolismo de los hidratos de carbono, proteínas, función osmótica, regulador del equilibrio ácido-básico intracelular y muchas otras.

En la diarrea se pierde una considerable cantidad de potasio. Según Darrow ${ }^{9}$ en una diarrea severa se pierden $10 \mathrm{mEq}$. por kilo de peso. Osea en un lactante de $5 \mathrm{Kgr}$. se perderán $50 \mathrm{mEq}$. $Y$ el contenido total de potasio es un lactante de $5 \mathrm{Kgr}$. (si consideramos un contenido de $40 \mathrm{mEq}$. por kilo de peso ${ }^{16}$ ), es de $200 \mathrm{mEq}$. Por lo tanto dicho paciente de $5 \mathrm{Kgr}$. de peso ha perdido el $25 \%$ de su contenido total de potasio.

Hasta hace poco tiempo se usaba el suero Ringer en la hidratación, habitualmente mezclado con suero glucosado. E1 suero Ringer contiene $4 \mathrm{mEq}$. de potasio por litro y el Ringer-glucosado, la mitad. Al hidratar a un lactante de $5 \mathrm{Kgr}$. con solución Ringer-glucosada, administrada en dosis de 200 cc. por kilo de peso, le damos sólo $2 \mathrm{mEq}$. de potasio. Salta a la vista la mínima cantidad de potasio si consideramos que el paciente ha perdido $50 \mathrm{mEq}$.

Según Darrow ${ }^{17}$ la sola administración de potasio en los sueros hidratantes descendió la mortalidad por toxicosis de un 30 a un $5 \%$.

En los últimos años se han propuesto varias soluciones ideales ${ }^{12}$, entre ellas la de Darrow que contiene $30 \mathrm{mEq}$. de potasio por litro.

Lo que se pretende con estas soluciones es reponer en la forma más exacta posible, todos los elementos que se han perdido: agua y electrólitos. Naturalmente que las pérdidas serán diferentes en cada paciente, dependiendo de la cuantía de la diarrea y vómitos. En vista de la imposibilidad de determinar el déficit en cada paciente debemos usar soluciones standard que nos den el máximun de garantias.

Con respecto a esto último, nos parece de sumo interés el detallado análisis reali- zado por Mönckeberg ${ }^{\top}$, del cual concluye que la dieta hidratante debe reunir las siguientes propiedades:

1. Proporcionar un adecuado aporte calórico en forma de hidratos de carbono.

2. Proporcionar una concentración electrolítica inferior a la del plasma.

3. Proporcionar una concentración electrolítica con predominio de valencias básicas, y

4. Proporcionar electrólitos tanto extra como intracelulares.

\section{ESQUEMA DE TRATAMIENTO}

En Noviembre de 1956 confeccionamos el siguiente esquema standard de tratamiento para los enfermos hospitalizados por diarrea aguda grave y toxicosis:

A. Tratamiento Antibiótico. Cloramfenicol por vía oral en dosis de 50 mgrs. por kilo de peso y fraccionado cada 6 horas, durante 7 días.

B. Tratd́miento Hidratante. Basándonos en el trabajo de Mönckeberg ${ }^{7}$, en el cual propone dos soluciones ideales para el tratamiento de la deshidratación; una para administrar por fleboclisis y otra por vía oral, usamos la última debido a la falta de elementos y personal preparado para administrarla por vía venosa.

La solución que usamos contiene:

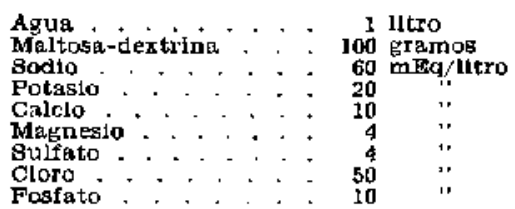

Como se puede apreciar dicha solución reúne las propiedades detalladas anteriormente. Administra $20 \mathrm{mEq} / \mathrm{L}$ de potasio, lo que no se obtenía anteriormente cuando administrábamos suero Ringer-glucosado. Aporta 400 calorías por litro, lo que permite prolongar sin riesgo la dieta hídrica en los distróficos.

La dieta hídrica duró de 24 a 48 horas, estando condicionada por la evolución de la enfermedad y el estado nutritivo del paciente. Se administró por gastroclisis a razón de 20 a 30 gotas por minuto. Previamente a la gastroclisis se practicó de rutina un lavado de estómago con suero fisiológico (por la misma sonda).

La cantidad de la dieta hídrica fué de $200 \mathrm{cc}$. por kilo de peso en las primeras 
24 horas, de las cuales la mitad se administró por gastroclisis. El volumen máximo dado por gastroclisis fué de $500 \mathrm{cc}$. En los casos en que se prolongó por más de 24 horas se siguió dando fraccionada cada una hora y con un total de $200 \mathrm{cc}$. por kilo de peso.

C. Tratamiento Dietético. El volumen de la dieta administrada a continuación de la dieta hidrica fué de 150 grs. por kilo de peso.

Durante los primeros dos días de realimentación se dió Eledón al $5 \%, 50$ grs. por kilo de peso, fraceionado cada 2 horas en 6 mamaderas. El Eledón se dió con crema de arroz. Los 100 grs. restantes (por kilo de peso), se dieron en la solución de maltosa-dextrina, en 12 veces, cada 2 horas y cubriendo las 24 horas.

Al tercero y cuarto día de realimentación se administró Eledón al $10 \%$, siempre en cantidad de $\mathbf{5 0}$ grs. por kilo de peso $\mathrm{y}$ en 6 dosis. Los 100 grs. restantes se administraron en forma de suero glucosado, fraccionado cada 2 horas. y 12 veces.

Además desde el tercer día se dió vitamina C 50 mgrs. diarios. Desde el cuarto día de realimentación en los mayores de 6 meses se dió sopa de zanahoria al $50 \%$.

Al quinto dia de realimentación se administró Eledón al $10 \%, 100$ grs. por kilo de peso, fraccionado en 6 mamaderas cada 3 horas y suero glucosado $50 \mathrm{cc}$, por kilo de peso, en dosis fraccionadas.

Al sexto día de realimentación se dió Eledón al $10 \%, 150$ grs. por kilo de peso cada 3 horas, 6 veces. Posteriormente se siguió según el criterio del médico tratante.

\section{MATERIAL}

Siguiendo el esquema de tratamiento anteriormente detallado, se trataron 41 lactantes con diarrea aguda grave, de los cuales $19(46,3 \%)$ presentaban como complicación el sindrome tóxico. Todos fueron pacientes hospitalizados en el Servicio de Pediatría del Hospital de Arica.

Ei primer paciente comenzó su tratamiento el 14 de Diciembre de 1956 y el último el 20 de Abril de 1957. Se llevó una hoja especial adjunta a la ficha clí- nica, con el objeto de anotar detalladamente Ja evolución de los diferentes síntomas $y$ signos, $y$ facilitar el estudio a posteriori.

a) Sexo.

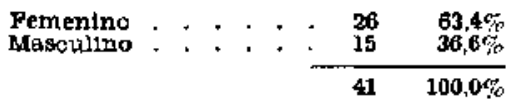

b) Edad.

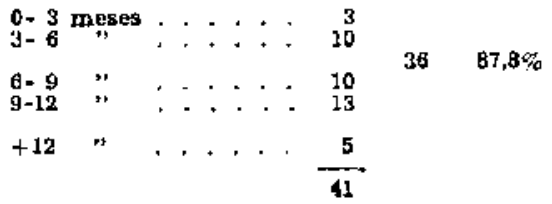

Como se puede apreciar la mayoría de nuestros pacientes $(87,8 \%)$, están comprendidos dentro del primer año de vida.

c) Estado nutritivo.

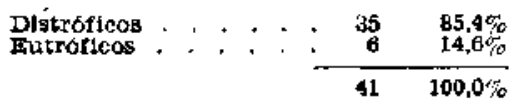

El estado nutritivo de nuestros pacientes era malo en $35(85,4 \%)$ de ellos. Es un hecho de todos conocido que la diarrea grave y toxicosis se presenta casi exclusivamente en pacientes distróficos. Coinciden nuestras cifras con las de Scroggie y Garcés ${ }^{13}$.

\section{d) Control anterior.}

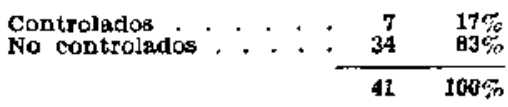

La gran mayoría de nuestros pacientes $(83 \%)$, no tenía un control pediátrico eficiente anterior a su enfermedad.

\section{RESULTADOS}

\section{A. Normalización del cuadro clínico.}

En el siguiente cuadro hemos colocado por grupos de pacientes según el día en que normalizaron los diferentes sintomas y signos. 
NORMALIZACION DE LOS STNTOMAS $Y$ BIGNOS

\begin{tabular}{|c|c|c|c|c|c|c|c|c|c|c|c|c|}
\hline$\cdot$ & ler. día & $29 \mathrm{~d}$. & 3er. d. & $49 \mathrm{~d}$. & $59 \mathrm{~d}$ & $60 \mathrm{~d}$. & $79 \mathrm{~d}$. & $89 \mathrm{~d}$. & $+8 \mathbf{d}$ & $\begin{array}{l}\text { No se mar- } \\
\text { malizó }\end{array}$ & $\begin{array}{l}\text { Normal } \\
\text { desdie } \\
\text { comlenzo }\end{array}$ & $\begin{array}{l}\text { Con } \\
\text { lavado } \\
\text { estom. }\end{array}$ \\
\hline $\begin{array}{l}\text { Dinrsea } \\
\text { Vomitos } \\
\text { Flebre } \\
\text { Obnubilacion } \\
\text { Hidratacion }\end{array}$ & $\begin{array}{l}1 \text { enf. } \\
3 \text { enf. } \\
3 \text { enf. } \\
9 \text { enf. }\end{array}$ & $\begin{array}{r}-1 \\
3 \\
19\end{array}$ & $\begin{array}{r}2 \\
1 \\
5 \\
12\end{array}$ & $\frac{5}{\frac{5}{2}}$ & $\begin{array}{r}6 \\
1 \\
10 \\
1\end{array}$ & $\frac{7}{5}$ & $\begin{array}{l}6 \\
- \\
-\end{array}$ & $\begin{array}{l}3 \\
- \\
-\end{array}$ & $\frac{4}{3}$ & $\begin{array}{l}7 \\
7 \\
7 \\
7 \\
7\end{array}$ & $\begin{array}{r}\overline{8} \\
1 \\
22 \\
\end{array}$ & 20 \\
\hline
\end{tabular}

a) Diarrea. Se normalizó dentro de los primeros 8 días de tratamiento en 30 pacientes $(73,2 \%)$. En cuatro pacientes $(9,7 \%$ ) se normalizó después de 8 días de tratamiento. En los 7 restantes $(17 \%)$, no se normalizó y fallecieron.

b) Vómitos. De los 33 pacientes que presentaban vómitos antes de iniciar el tratamiento, en $20(60 \%)$, no se presentó después del lavado de estómago previo a la gastroclisis. Conviene hacer notar que con el lavado de estómago practicado al ingresar el paciente al Servicio, se extrae del estómago en la mayoría de ellos, un líquido café oscuro o negro, de mal olor y a veces con sangre. Nos impresiona que el solo hecho de eliminar del estómago este líquido con toda seguridad cargado de toxinas, contribuye en gran parte a suspender los vómitos.

c) Fiebre. La temperatura se normalizó dentro de los 7 primeros días de tratamiento en $30(75 \%)$ de los 40 pacientes que presentaban fiebre al ingreso.

d) Obnubilación. De los 19 pacientes con sindrome tóxico, el sensorio se normalizó dentro de los 2 primeros días de tratamiento en 12 de ellos. Los 7 restantes fallecieron.

e) Hidratación. La normalización se apreció clínicamente por el examen de la piel, mucosas, globo ocular, fontanela, etc. Se normalizó dentro de los 5 primeros días de tratamiento en 34 pacientes $(83 \%)$. Los 7 restantes fallecieron.

En algunos pacientes hubo necesidad de administrar una segunda gastroclisis.

\section{B. Complicaciones.}

Dos pacientes presentaban Bronconeumonía al ingreso. Se trataron además del esquema con Penicilina y Estreptomicina parenteral. Mejoraron de su complicación pulmonar al $3^{\circ}$ y $4^{\circ}$ día de tratamiento respectivamente.

Ocho pacientes presentaron hiperpirexia mantenida durante los primeros días de tratamiento. En cuatro de ellos se normalizó con tratamiento sintomático. I os otros cuatro fallecieron.

Conviene hacer notar que hemos obser- vado en la mayoría de nuestros pacientes un alza térmica al instalar la gastroclisis, la cual se mantiene durante algunas horas. Pensamos que puede influir en este fenómeno el traumatismo producido por la sonda y la absorción de toxinas por la mucosa digestiva.

Un paciente presentó convulsiones durante el primer dia asociado a hiperpirexia. Cedieron con tratamiento sintomático.

\section{Letalidad.}

De los 41 pacientes fallecieron 7 (17\%). En 6 de ellos no hubo ninguna respuesta al tratamiento.

Uno falleció a las 36 horas de tratamiento, otro al $2^{\circ}$ día, tres al 3er. dia y dos al $6^{\circ}$ día.

Otro paciente respondió satisfactoriamente al tratamiento durante los primeros 7 días. Al $8^{?}$ día presentó glositis y estomatitis ulcerosa. Al $10^{\circ}$ día apareció un exantema máculo-papuloso escasamente confluente y generalizado. Al $12^{\circ}$ día reapareció la diarrea y fiebre. Se agravó progresivamente y fallece al $16^{\circ}$ día.

\section{Dias de hospitalización.}

El promedio fué de 10,4 días. Esto se debe a que la mayoría de nuestras pacientes $(\mathbf{8 5}, 4 \%)$ eran distróficos $\mathrm{y}$ hubo que reparar parcialmente su estado nutritivo antes de darlos de alta.

\section{COMENTARIO}

El problema de ia diarrea grave y su alta mortalidad seguirá siendo igual mientras no contemos con lactantes de mejor calidad. Se puede decir que la diarrea no es la causa de muerte, sino la distrofia. El $85 \%$ de nuestros pacientes eran distróficos. Por otra parte el $83 \%$ no se había controlado en forma eficiente. Esto último es un factor de mucha importancia en la producción de distrofias.

La mortalidad nuestra de $17 \%$ se puede considerar baja, si se toma en cuenta que no contamos en forma rutinaria con elementos terapéuticos de gran ayuda; 
plasmoterapia, transfusiones, fleboclisis, etc.

Nos parece de interés recalcar la importancia de usar soluciones hidratantes con un adecuado contenido de potasio. La solución propuesta por Mönckeberg ${ }^{?}$ que teóricamente reúne todos los requisitos para tratar la deshidratación aguda, en la práctica nos ha dado excelente resultado.

La hidratación por gastroclisis da muy buenos resultados, pero en aquellos enfermos graves que continuan con diarrea y vómitos intensos es más aconsejable re currir a la fleboclisis.

El lavado de estómago previo a la gastroclisis nos parece de mucha importancia, contribuyendo a suprimir el vómito.

Conviene destacar la importancia de seguir un esquema standard de tratamiento para una enfermedad frecuente en la época de verano. En esta forma se facilita enormemente el trabajo del médico y personal auxiliar. Por lo demás, en hospitales generales donde no hay Pediatraresidente, el cirujano de turno puede indicar con más facilidad el tratamiento guiándose por el esquema.

\section{CONCLUSIONES}

1. Se destaca la importancia de la diarrea como una de las principales causas de mortalidad infantil en Chile.

2. Se analiza el tratamiento de la diarrea grave y toxicosis del lactante destacándose principalmente en el tratamiento hidratante, la importancia de administrar soluciones con un contenido adecuado de potasio, debido a la gran pérdida de este electrólita por las deposiciones. Se anotan las propiedades que según Mönckeberg debe reunir una solución hidratante ideal.

3 . Se resume detalladamente el esquema de tratamiento usado en el Servicio de Pediatría del Hospital de Arica para las diarreas graves y toxicosis.

Se analiza la solución hidratante propuesta por Mönckeberg para ser usada por vía oral. Se destaca el contenido de potasio $(20 \mathrm{mEq} / \mathrm{L}\}$ y su alto contenido energético.

4. El material consta de 41 pacientes con diarrea aguda grave, de los cuales 19 $(46,3 \%)$ presentaban sindrome tóxico. Treinta $y$ seis $(87,8 \%)$ eran lactantes menores de un año de edad. Treinta $\mathrm{y}$ cinco $(85,4 \%)$ eran distróficos y 34 $(83 \%)$ no tenian un control pediátrico eficiente anterior a su enfermedad.
5. La diarrea se normalizó dentro de los primeros 8 días de tratamiento en 30 pacientes $(73,2 \%)$. Los vómitos no se presentaron después del lavado de estómago en $20(60 \%)$ de los 33 pacientes que lo presentaban al ingreso. La fiebre se normalizó dentro de los siete primeros días de tratamiento en $30(75 \%)$ de los 40 pacientes que presentaban este sintoma al iniciar el tratamiento. La hidratación se normalizó dentro de los 5 primeros dias de tratamiento en $34(83 \%)$ pacientes.

6. Se observó un alza térmica durante la hidratación con gastroclisis en la mayoría de nuestros pacientes.

7. De los 41 pacientes fallecieron 7 (17\%). En 6 de ellos no hubo ninguna respuesta al tratamiento.

8. Estos resultados pueden considerarse como un éxito, ya que no contamos en forma rutinaria con otros elementos de gran ayuda tales como la plasmoterapia, transfusiones y fleboclisis.

\section{RESUMEN}

Se exponen los resultados obtenidos en el Servicio de Pediatría del Hospital de Arica en el tratamiento de la Diarrea Aguda grave $\mathrm{y}$ Toxicosis en 41 lactantes a los cuales se les aplicó un esquema común de tratamiento.

\section{SUMMARY}

TREATMENT OF SEVERE ACUTE DIARHHEA AND TOXICOSIS.

A statement is made on the results obtained in the Pediatric Department of the Arica Hospital in the treatment of severe Acute Diarrhea and Toxicosis in 41 infants whom receibed a standard plan of treatment.

\section{BIBLIOGRAFÍA}

ג.-MeneatelLo, J. - VII Congreso Nacional de Pedintría, 1956.

2.-Defunctones $y$ Causas de Muerte en la República de chlle. Servacio Nactonal de Salud. 1955.

3. -BRAND, $G$. - Por publjcarse.

4.-COSTA. A. - Revista Chllena de Ped. $22: 233,1956$.

5.-RODFIC UiEz, M. - Revista Chilena de Ped. 27: 197,1956 .

6.一WHEELRR, W. E. y col. - Am. J. Dis. Child. 86 : 350.1953.

7.-MONCKFBERG, F. - Revista Chllene de Ped. 27: 268,1956 .

8.-GAzMuRI. R. - Eevista Médica de Chile. 83:381, 1955.

9.-DARROW. D. C. y col. - Pediatrics. 3:129. 1942.

10.-CORzA $y$ col. - C1tado por Gazmurl, $R$. Rev. Médlca de Chile. B3:381, 1955

11. GOVAN, C, D, $y$ DARkOW. D. C. - J, Pediat, 28: 541, 1946.

12.-Wrisberg, H. F. - Metabolismo del Agua $y$ de los Electrólitos. (Editorla) Artécnica). 1954.

13.-SCROGGIE, A, y GARCES, H, - Revista Chllen de Ped, 24:319, 1953. 